\title{
Les malocclusions
}

\section{et les traitements orthodontiques sont-ils responsables de l'apparition de désordres temporo-mandibulaires?}

\section{RÉSUMÉ}

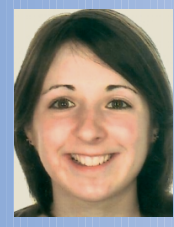

Julie LAVIGNASSE

Docteur en chirurgie dentaire,

1, rue Godefroy,

75013 Paris.

Magaly VERTHIER

Assistante hospitalo-universitaire,

Faculté de chirurgie dentaire,

Paris V.

Alain LAUTROU

Professeur des Universités, Praticien hospitalier,

Faculté de chirurgie dentaire,

Paris V.
Les désordres temporo-mandibulaires rassemblent tous les problèmes cliniques concernant les muscles masticateurs et/ou l'articulation temporo-mandibulaire. Ils ont une origine multifactorielle et la responsabilité des malocclusions et des traitements orthodontiques est actuellement très contestée. Cet article, à travers une revue de la littérature, tente d'éclaircir les données actuelles.
- désordres temporo-mandibulaires

- malocclusions

- orthodontie 


\section{Les malocclusions}

\section{et les traitements orthodontiques} sont-ils responsables de l'apparition
de désordres temporo-mandibulaires?

$>$

Les désordres temporo-mandibulaires (DTM) sont regroupés sous un terme générique rassemblant les problèmes cliniques concernant les muscles masticateurs et/ou I'articulation temporo-mandibulaire. La prévalence des signes et des symptômes de ces pathologies augmente lors de l'adoles- cence, période pendant laquelle un traitement orthodontique est souvent mis en place. Cet article tente d'apporter des réponses à travers une revue de la littérature sur le rôle étiologique potentiel des malocclusions et de I'un de ses traitements correcteurs, I'orthodontie.

\section{Responsabilité des malocclusions}

Les malocclusions sont historiquement les facteurs primaires responsables du développement de désordres temporo-mandibulaires. Cependant, en 1996, dans un rapport du NIH Technology Assessment Conference on Management of Temporomandibular Disorders [1], de nombreux chercheurs, scientifiques et cliniciens présentèrent les résultats de leurs études (mises en œuvre selon des critères de recherche internationalement acceptés) portant sur l'étiologie occlusale des désordres temporomandibulaires. La confusion fut grande car les résultats étaient contradictoires; les scientifiques diminuaient l'importance des facteurs occlusaux et les praticiens continuaient d'adhérer au concept d'étiologie occlusale des DTM et à corriger les malocclusions pour traiter ces pathologies. Actuellement, il est communément admis que seuls cinq facteurs occlusaux seraient significativement associés aux désordres temporo-mandibulaires [14] :
- une béance antérieure d'origine squelettique :

- un surplomb supérieur à 6-7 mm ;

- une différence entre la relation centrée et l'occlusion inter-maxillaires supérieure à $4 \mathrm{~mm}$;

- une occlusion inversée unilatéralement ;

- la perte de 5 dents postérieures ou plus.

Depuis cet article de Mac Namara et al., de nombreuses études ont analysé des facteurs occlusaux (I'encombrement dentaire, l'excès ou I'insuffisance de surplomb et de recouvrement incisifs, le décalage des milieux interincisifs, les interférences occlusales...) mais il existe à ce jour peu de consensus; beaucoup d'études se contredisent du fait de l'absence d'une définition commune aux désordres temporo-mandibulaires, du caractère transitoire de ces pathologies et de leur origine multifactorielle (anatomique, physiopathologique, psychologique, traumatique) qui rendent les analyses statistiques d'un facteur isolé difficiles à réaliser. 


\section{Responsabilité}

\section{des thérapeutiques orthodontiques}

Actuellement, le rôle des traitements orthodontiques dans l'apparition de désordres temporo-mandibulaires n'a pas encore été prouvé et ces thérapeutiques n'augmenteraient pas le risque de développer ces pathologies $[4,6,8,15,18,20]$ même si l'occlusion idéale n'est pas atteinte en fin de traitement [10, 14]. II n'y aurait aucune augmentation de la prévalence des signes et des symptômes de DTM chez le patient ayant suivi un traitement orthodontique $[7,10,12,18]$. De plus, les thérapeutiques orthodontiques ne constitueraient pas un facteur étiologique important [12] ou significatif [15] et ne seraient pas corrélées aux signes et symptômes de DTM $[2,16]$. La récidive, après une thérapeutique orthodontique précoce, n'augmenterait pas le risque de développer des signes et symptômes de DTM chez les patients jeunes [19]. II n'existerait aucune différence

\section{Conclusion}

Les malocclusions et les traitements orthodontiques seraient des cofacteurs à une mosaïque de problèmes multifactoriels et, seuls, n'augmenteraient pas le risque de développer un désordre temporo-mandibulaire et ce grâce à la possibilité d'adaptation et de compensation entre les traitements avec ou sans extractions concernant l'étiologie des DTM $[2,11,13,14$, 18] et aucune thérapeutique fixe, extra-orale ou orthognathique n'entraînerait l'apparition de ces pathologies $[2,3,5,9,17]$.

Le rôle préventif des thérapeutiques orthodontiques est toujours contesté : un traitement orthodontique quel qu'il soit ne diminuerait pas le risque de développer un DTM $[10,15,20]$. Face à un sujet sain, le praticien devra veiller à respecter en toute logique les règles d'équilibre occlusal et individualiser ses traitements. La classe I d'Angle est choisie pour sa stabilité mais, par exemple, il est parfois préférable de terminer le traitement en classe II thérapeutique bien équilibrée plutôt qu'en classe I imparfaite. La connaissance des règles occlusales et le bon sens clinique sont indispensables car il existe peu de certitudes.

individuelle. Il existe de nombreuses interrelations entre les DTM et d'autres domaines (rhumatologie, ORL, odontologie, neurologie, psychologie...) et, pour mieux les traiter, le patient doit être pris en charge par une équipe pluridisciplinaire. 


\section{Bibliographie}

1. De Boever JA, Carlsson GE, Klineberg IJ.

Need for occlusal therapy and prosthodontic treatment

in the management of temporomandibular disorders. Part I.

J Oral Rehabil 2000;27(5):367-379.

2. Dibbets JM, Van Der Weele LT. Long-term effects of orthodontic treatment, including extraction, on signs and symptoms attributed to TMD. Eur J Orthod 1992; 14(1):16-20.

3. Egermark I, Blomquist JE, Cromvisk U, et al. Temporomandibular dysfunction in patients treated with orthodontics in combination with orthognathic surgery. Eur J Orthod 2000;22(5):537-544.

4. Egermark I, Magnusson T, Carlsson GE.

A 20-year follow-up of signs and symptoms of temporomandibular disorders and malocclusions in subjects with and without orthodontic treatment in childhood.

Angle Orthodontist 2003;73(2):109-115.

5. Franco AA, Yamashita HK, Lederman HM, et al. Fränkel appliance therapy and the temporomandibular disc: a prospective magnetic resonance imaging study.

Am J Orthod Dentofacial Orthop 2002;121(5):447-457.

6. Henrikson $\mathrm{T}$, Nilner $\mathrm{M}$, Kurol J.
Temporomandibular disorders and the need for stomatognathic treatment in orthodontically treated and untreated girls. Eur J Orthod 2000;22(3):283-292.

7. Henrikson T, Nilner M. Temporomandibular disorders, occlusion and orthodontic treatment. $\mathrm{J}$ of Orthod 2003;30(2):129-137.

8. Hirata $\mathrm{RH}, \mathrm{Heft} \mathrm{MW}$, Hernandez B, et al. Longitudinal study of signs and symptoms of signs of temporomandibular disorders (TMD) in orthodontically treated and non treated groups. Am J Orthod Dentofacial Orthop 1992;101(1):35-40.

9. Keelings SD, Garvan CW, King GJ, et al.

Temporomandibular disorders after early

Class II treatment with bionators and headgears: results from a randomized controlled trial.

Semin Orthod 1995;1(3):149-164.

10. Kim MR, Graber T,

Viana MA.

Orthodontics

and temporomandibular disorders:

a meta-analysis.

Am J Orthod

Dentofacial Orthop 2002;121(5):438-446.

11. Kremenak $C R$, Kinser $D D$, Harman HA, et al. Orthodontics risk factor for temporomandibular disorders I.

Am J Orthod Dentofacial Orthop 1992;101(1):13-20.
12. Kremenak $C R$, Kinser $D D$, Melcher TJ, et al.

Orthodontics as a risk factor for temporomandibular disorders II.

Am J Orthod Dentofacial Orthop 1992;101(1):21-27.

13. Kundinger $\mathrm{KK}$, Austin $\mathrm{BP}$, Christensen LV, et al.

An evaluation

of temporomandibular joints and jaw muscles after orthodontic treatment, involving premolar extractions.

Am J Orthod Dentofacial Orthop 1991;100(2):110-115.

14. Mac Namara JA, Seligman DA, Okeson P. Occlusion, orthodontic treatment and temporomandibular disorders: a review. J Orofac Pain 1995; 9(1): 73-90.

15. Mohlin BO, Derweduwen $\mathrm{K}$, Pilley R, et al.

Malocclusion and temporomandibular disorder: a comparison of adolescents with moderate to severe dysfunction with those without signs and symptoms of temporomandibular disorder and their further development to $\mathbf{3 0}$ years of age.

Angle Orthod 2004;74(3):319-327.

16. Rendell JK, Norton LA, Gay T.

Orthodontic treatment and temporomandibular joint disorders.

Am J Orthod Dentofacial Orthop 1992;10(1):84-87.

17. Ruf $S$, Pancherz $H$. Does bite-jumping 
damage the TMJ?

A prospective longitudinal clinical and MRI study of Herbst patients. Angle Orthod 2000;70(3):183-199.

18. Sadowsky C, Thiesen TA, Sakols EI.

Orthodontic treatment and temporomandibular joint sounds: a longitudinal study.
Am J Orthod Dentofacial Orthop 1991;99(5):441-447.

19. Tullberg $M$, Tsarapatsani $P$, Huggare J, et al. Long term follow up of early treatment of unilateral forced cross bite with regard to temporomandibular disorder and associated symptoms.

Acta Odontol Scand
2001;59(5):280-284.

20. Wadhwa L, Utreja A, Tewari A.

A study of clinical signs and symptoms of temporomandibular dysfunction in subjects with normal occlusion, untreated and treated malocclusions.

Am J Orthod Dentofacial Orthop 1993;103(1):54-61.

\section{SUMMARY}

\section{Are malocclusions and orthodontic treatments} responsible of the occurrence

\section{of temporomandibular disorders?}

Julie LAVIGNASSE,

Magaly VERTHIER,

Alain LAUTROU

Keywords

- temporomandibular disorders

- malocclusions

- orthodontic treatment
Temporomandibular disorders include all the clinical problems of jaw muscles and/or temporomandibular joint. Multifactorial aetiology is recognized and the role of malocclusions and orthodontic treatments is at present time disputed.

This article, through a review of the literature, tries to clarify the present datas.

\section{Questions - Réponses}

1/ Réponse par vrai ou faux

a - Les traitements orthodontiques sont considérés comme une étiologie des DTM
Vrai
Faux

b - Il existe une différence pour l'apparition des DTM chez les patients traités orthodontiquement avec ou sans extractions
Vrai
Faux

xnef: q - xnef: e : səsuodöy 


\section{WW W. G P S V O . N E T}

recteur n' 1 d'ingtabilitís

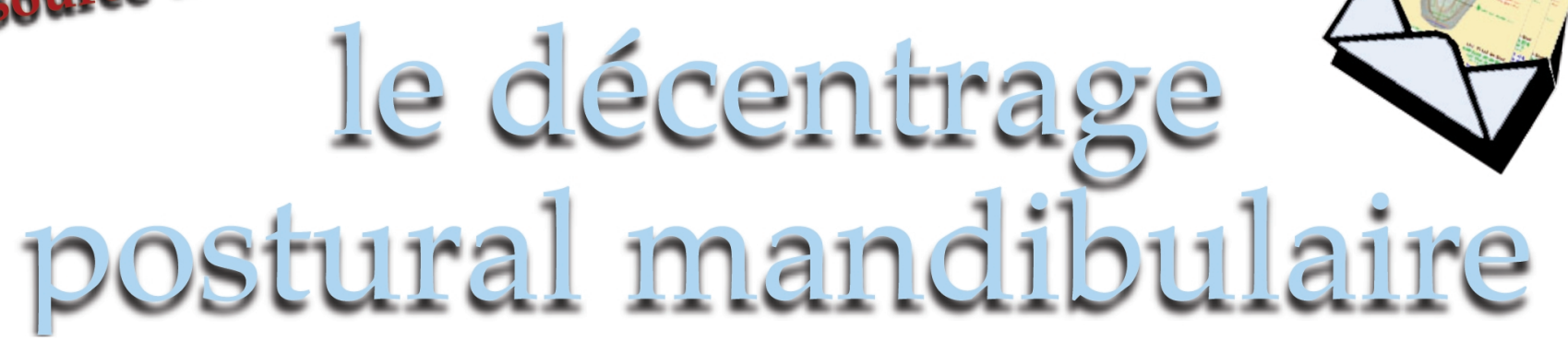

comment vérifier la posture mandibulaire en 3D en occlusion statique?

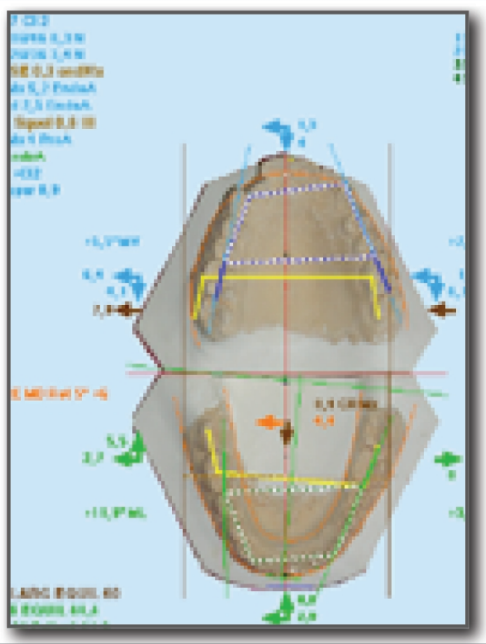

\section{AVEC \\ L' ANALYSE GPSvo}

par Géo - Positionnement des Structures

diagnostic étiologique et

différentiel des asymétries

comment corriger un décentrage postural mandibulaire?

par

\section{LA TECHNIQUE ROO}

Réhabilitation Occluso - posturale Orthodontique avec les Ancreurs ${ }^{\circledast}$ de ROO

selon une mécanique 3D indépendante d'un hémi-quadrant à l'autre

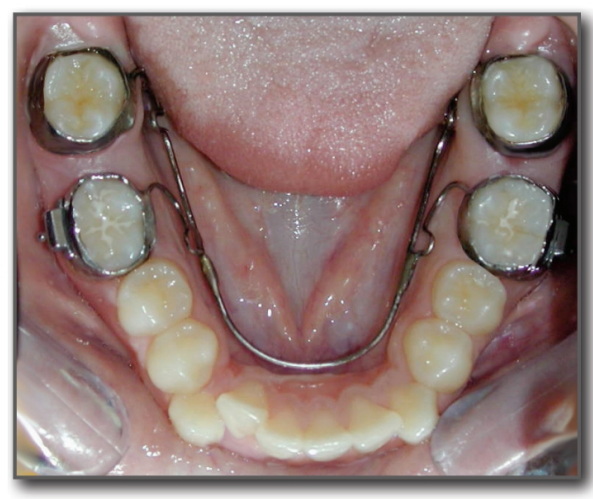

\section{FORMATIONS A LA GARTE par le Dr J. GHOUZI DUVAL}

\section{SÉMINAIRES au choix \\ DE PRATIQUE \\ ou

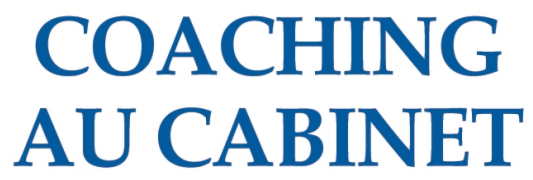

RENSEIGNEMENTS SUR LES FORMATIONS SUR LE SITE ET PAR MAIL CONTACT POUR LES ANALYSES ET LES ANCREURS DE ROO

wwww.gpsvo.net ORTHOFORMACCESS Tél : 0625817010 contact@gpsvo.net 